\title{
AGILE E-BUSINESS PROCESS ASSEMBLY AND DEVELOPMENT
}

\author{
Thomas Schmidt, Karl Fürst, Gerald Wippel \\ Automation Control Institute - TU Vienna \\ ts@infa.tuwien.ac.at,kf@infa.tuwien.ac.at,gw@infa.tuwien.ac.at
}

\begin{abstract}
A collaborative enterprise uses Internet technology to achieve dramatic improvements along the whole life cycle by integrating the business processes of collaborating partners. Therefore it is necessary to describe the public aspects of business processes in a standard way using international standardized languages like WSFL, XLANG, BPML, or ebXML. In this paper the architecture for automatically program code generation to realize the business process tier of the FLoCI-EE system architecture is discussed. This tier uses feature rich business Web Services to realize business processes as a composition of Web Services and provide the business processes as Web Services.
\end{abstract}

\section{INTRODUCTION}

The ultimate goal of companies is the delivery of high quality products and services to the global market within the shortest possible time (time-to-market) and lowest costs (time-to-money) in order to be competitive. Because more and more enterprises focus on their core-competencies, they have to collaborate to support the whole life cycle of complex products or services. Such collaborations are temporary or permanent networks of independent enterprises that cooperate with the aim to design, manufacture, and sell a product or service independent of enterprise borderlines. Each member contributes specific core know-how to the collaboration network. This concept enables very fast and flexible reactions to changed market conditions or new opportunities.

Enterprise Application Integration (EAI) follows the aim to integrate isolated application implementations among each other or with central and enterprise-wide systems like ERP. Such realized integration solutions are often selective and restricted application integration approaches which means that data-oriented oneway integration solutions without synchronization of the underlying business processes stand in the foreground (Linthicum, 2000). To support the integration of enterprise-wide and enterprise-spanning business processes, we need the next step in the EAI-evolution: the process-centric EAI called business integration. 


\section{BUSINESS INTEGRATION}

With the growth of e-commerce there is an emerging need to integrate and automate business processes that span enterprise boundaries (the so-called B2C and B2B processes). A business process can be defined as a collection of business transactions between partners and/or internal activities within one business. A typical business process may consist of up to 100 IT transactions. Coordinating the entire process correctly and efficiently places severe demands on the organization's IT infrastructure.

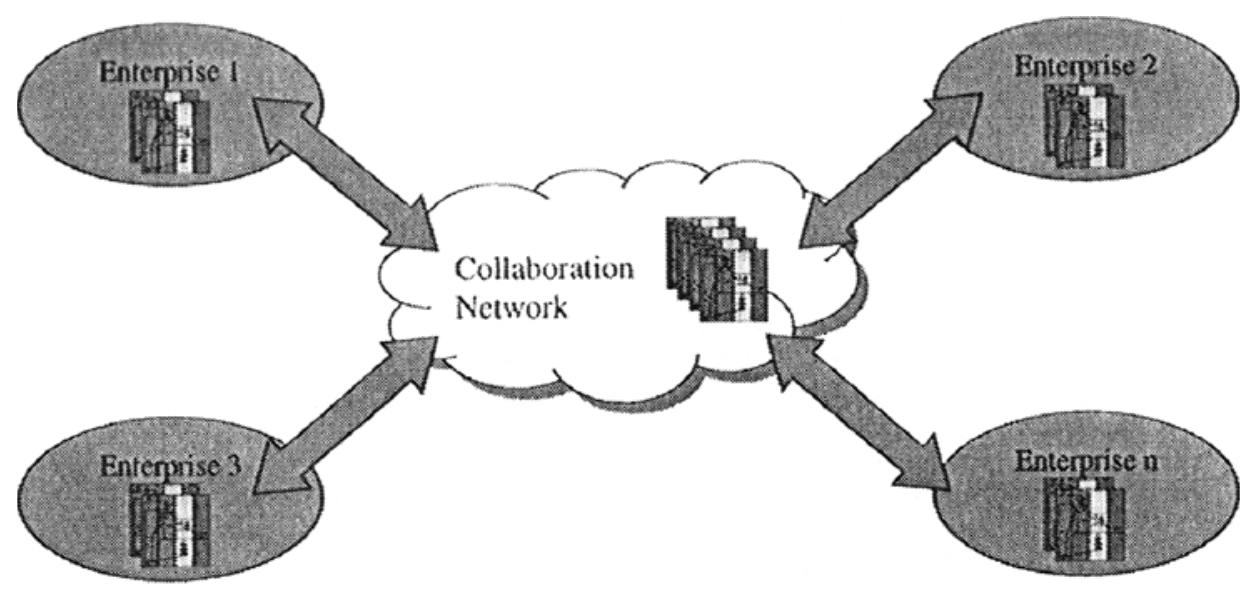

Figure 1 - Business Processes in the Collaboration Network

Figure 1 shows the collaboration network scenario with internal enterprise specific and common business processes. All processes are networked to achieve the common goal of delivering high quality products and services to the customers. Each enterprise inside the network has their own core-competencies like engineering, production, or marketing and sales. Members of the collaboration network are also the suppliers with the core-competence to deliver specific raw materials and semi-finished goods. A tight integration of the customers is needed especially in the case of creation complex products and services.

The currently running international research and development project FLoCI-EE (Flexible Low-Cost Internet Extended Enterprise) aims at the development of an easy to use, component based software system to support the whole lifecycle of products in extended enterprises (Fürst, 2001). Extended enterprises are special forms of collaboration networks where one dominant enterprise "extends" its boundaries to all or some of its suppliers. During this project, more precisely at architecture design, the need for a separate business process tier was detected (see Figure 2). This tier uses feature rich business Web Services (e.g. Document Management, Project Management, etc.), which are provided by the lower tier, to realize business processes as a composition of Web Services. These business processes are provided as Web Services to the upper tier. 


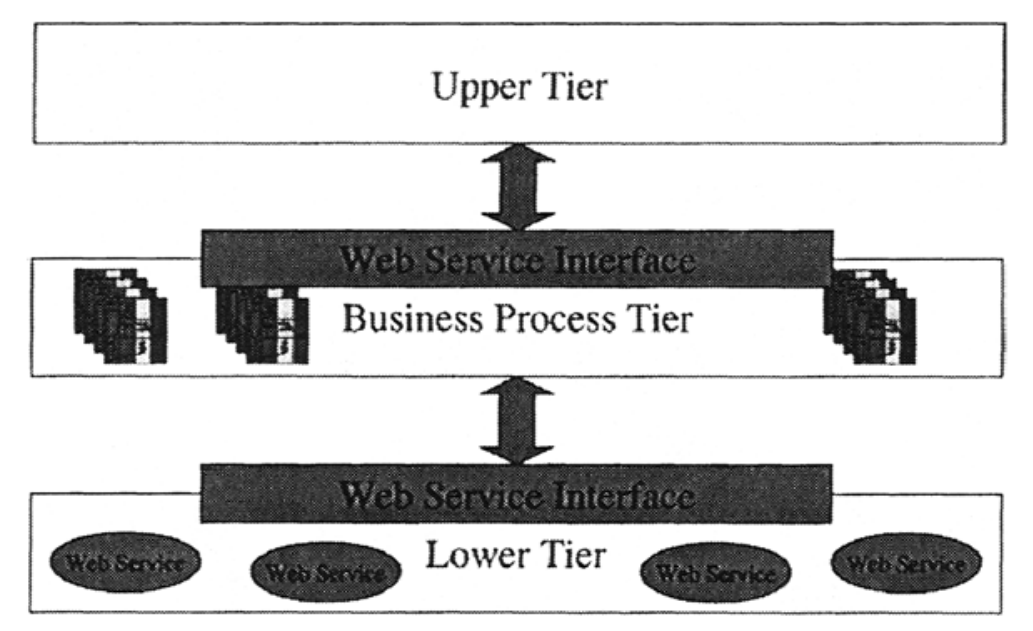

Figure 2 - Business Process Tier

The following chapter gives a short introduction into the up-to-date topic Web Services. In chapter 4 the current available Web Service based business process modeling standards WSFL, XLANG, and BPML are discussed. Additionally mentioned is the promising standard ebXML. In chapter 5 the vision of automatically program code generation using the description of the business processes is described.

\section{WEB SERVICES}

A Web Service represents a unit of business, application, or system functionality that can be accessed over the Web. Web Services are applicable to any type of Web environment, whether Internet, Intranet, or Extranet, whether with a focus on business-to-consumer, business-to-business, department-to-department, or peer-topeer communication. A Web Service consumer could be a human user accessing the service through a desktop or wireless browser; it could also be an application program or another Web Service (Sun, 2002).

A Web Service exhibits the following basic characteristics:

- Web Services communicate using XML messages over standard Web protocols. SOAP (Simple Object Access Protocol) provides an extensible XML messaging protocol and also supports an RPC (Remote Procedure Call) programming model (SOAP, 2000). An extended variant of SOAP, called SOAP Messages with Attachments, is using MIME to be able to transport also non XML-payload.

- A Web Service exposes an XML interface description. WSDL (Web Service Description Language) is a common XML framework for describing a Web Service (WSDL, 2001).

- A Web Service is registered and can be located through a Web Service registry. The UDDI (Universal Description, Discovery, and Integration) initiative is an industry consortium that is developing specifications for 
a universal, Web-based business directory called the UDDI Business Registry (UDDI, 2002).

Figure 3 shows the cooperation between a service provider, who has published the Web Service at a service registry, and a service requestor, who has found the Web Service at this service registry.

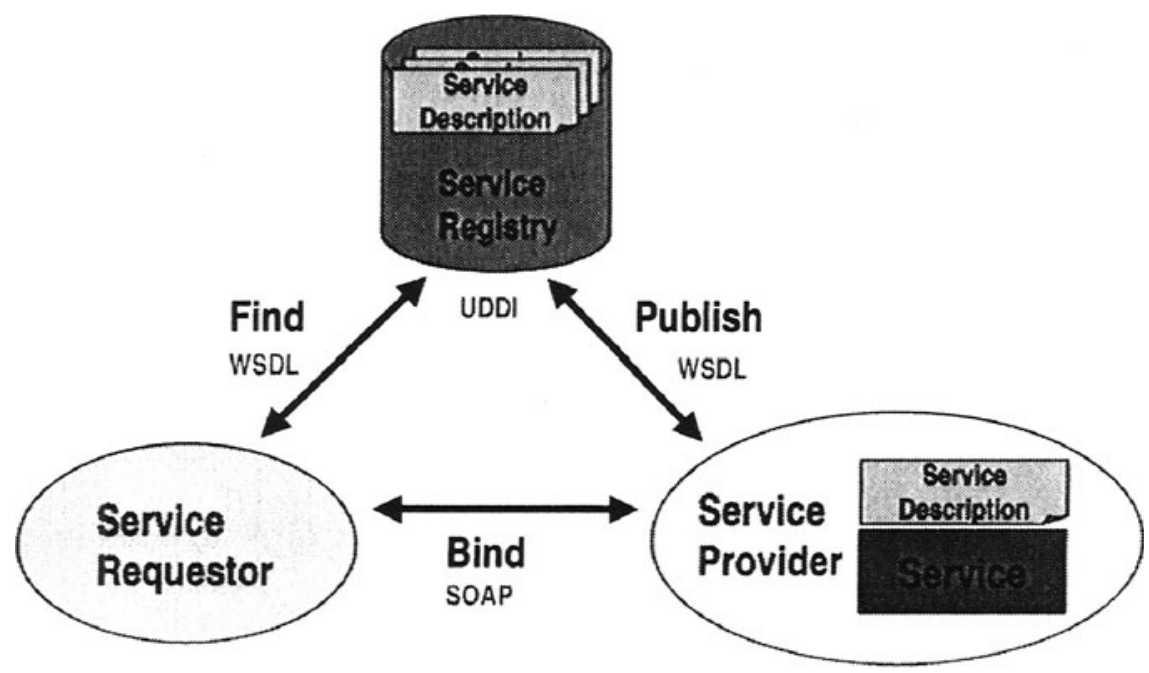

Figure 3 - Web Service Model

\section{BUSINESS PROCESS MODELING}

One precondition for enabling cross-enterprise business process automation is the ability to describe the public aspects of the business processes in a standard form that can be consumed by tools for process implementation and monitoring. In the following subchapters, the currently available XML-based approaches to fulfill this condition are discussed.

\subsection{WSFL}

The WSFL (Web Service Flow Language) is an XML language for the description of Web Services compositions as part of a business process definition. It was designed by IBM to be part of the Web Service technology framework and relies and complements existing specifications like SOAP, WSDL, and UDDI (WSFL, 2001).

WSFL considers two types of Web Service compositions:

- The first type specifies an executable business process known as a flow model.

- The second type specifies a business collaboration known as a global model.

In the first case, a composition is created by describing how to use the functionality provided by the collection of composed Web Services. This is also known as flow composition, orchestration, or choreography of Web Services. WSFL 
models these compositions as specifications of the execution sequence of the functionality provided by the composed Web Services. Execution orders are specified by defining the flow of control and data between Web Services. Flow models can especially be used to model business processes or workflows based on Web Services.

In the second case, no specification of an execution sequence is provided. Instead, the composition provides a description of how the composed Web Services interact with each other.

WSFL provides extensive support for the recursive composition of services: In WSFL, every Web Service composition (a flow model as well as a global model) can itself become a new Web Service, and can thus be used as a component of new compositions.

\subsection{XLANG}

XLANG is the XML business process language used by Microsoft's BizTalk server. The goal of XLANG is to make it possible to formally specify business processes as stateful long-running interactions. Business processes always involve more than one participant. The full description of a process must show not only the behavior of each participant, but the way these behaviors match to produce the overall process. The focus is on the publicly visible behavior in the form of message exchanged. The specific high-level feature categories that define the scope of XLANG are listed below (XLANG, 2002):

- Sequential and parallel control flow constructs

- Long running transactions with compensation

- Custom correlation of messages

- Flexible handling of internal and external exceptions

- Modular behavior description

- Dynamic service referral

- Multi-role contracts

XLANG has a close relationship with WSDL: An XLANG service description is a WSDL service description with an extension element that describes the behavior of the service as a part of a business process.

Microsoft has previously worked with IBM on both the WSDL and UDDI initiatives. And, while they have been initially creating parallel recommendations, it would not be surprising to see IBM and Microsoft jointly agree to submit a proposal to W3C that combines XLANG and WSFL in the near future.

\subsection{BPML}

The BPML (Business Process Modeling Language) is a meta-language for the modeling of business processes, just as $\mathrm{XML}$ is a meta-language for the modeling of business data (BPML, 2001). BPML provides an abstracted execution model for collaborative and transactional business processes based on the concept of a transactional finite-state machine.

The BPML specification is provided by the business process management initiative (BPMI) organization. BPMI.org is a non-profit corporation that empowers companies of all sizes, across all industries, to develop and operate business 
processes that span multiple applications and business partners, behind the firewall and over the Internet. The initiative's mission is to promote and develop the use of Business Process Management (BPM) through the establishment of standards for process design, deployment, execution, maintenance, and optimization.

In much the same way XML documents are usually described in a specific XML Schema layered on top of XML, BPML processes can be described in a specific business process modeling language layered on top of the extensible BPML XML Schema. BPML represents business processes as the interleaving of control flow, data flow, and event flow, while adding orthogonal design capabilities for business rules, security roles, and transaction contexts.

In addition, the BPMI is also working on a business process query language (BPQL), which will provide the interface between the application and the business process data. The idea is that BPQL will eventually be to BPML what SQL is to databases.

\section{4 ebXML}

To provide an open XML-based infrastructure enabling the global use of electronic business information in an interoperable, secure and consistent manner by all parties is the mission of ebXML (Electronic Business using XML). ebXML, sponsored by UN/CEFACT (United Nations body for Trade Facilitation and Electronic Business Information Standards) and OASIS (Organization for the Advancement of Structured Information Standards), is a modular suite of specifications that enables enterprises of any size and in any geographical location to conduct business over the Internet. Using ebXML, companies now have a standard method to exchange business messages, conduct trading relationships, communicate data in common terms and define and register business processes (ebXML, 2001).

The following overview introduces the concepts and underlying architecture of ebXML:

- A standard mechanism for describing a business process and its associated information model.

- A mechanism for registering and storing business process and information meta-models so they can be shared and reused.

- Discovery of information about each participant including:

- The business processes they support.

- The business service interfaces they offer in support of the business process.

- The business messages that are exchanged between their respective business service interfaces.

- The technical configuration of the supported transport, security and encoding protocols.

- A mechanism for registering the aforementioned information so that it may be discovered and retrieved.

- A mechanism for describing the execution of a mutually agreed upon business arrangement which can be derived from information provided by each participant. 
- A standardized business messaging service framework that enables interoperable, secure and reliable exchange of messages between trading partners.

- A mechanism for configuration of the respective messaging services to engage in the agreed upon business process in accordance with the constraints defined in the business arrangement.

\section{CONCLUSION AND FUTURE WORK}

In chapter 4 the state-of-the-art in XML-based business process modeling was discussed. The future progress of standardization efforts will show if the different modeling languages will grow together to establish only one XML-based modeling language.

To realize the vision of automatically program code generation using the XMLbased description of the business processes in a standardized form, the architecture shown in Figure 4 will be used.

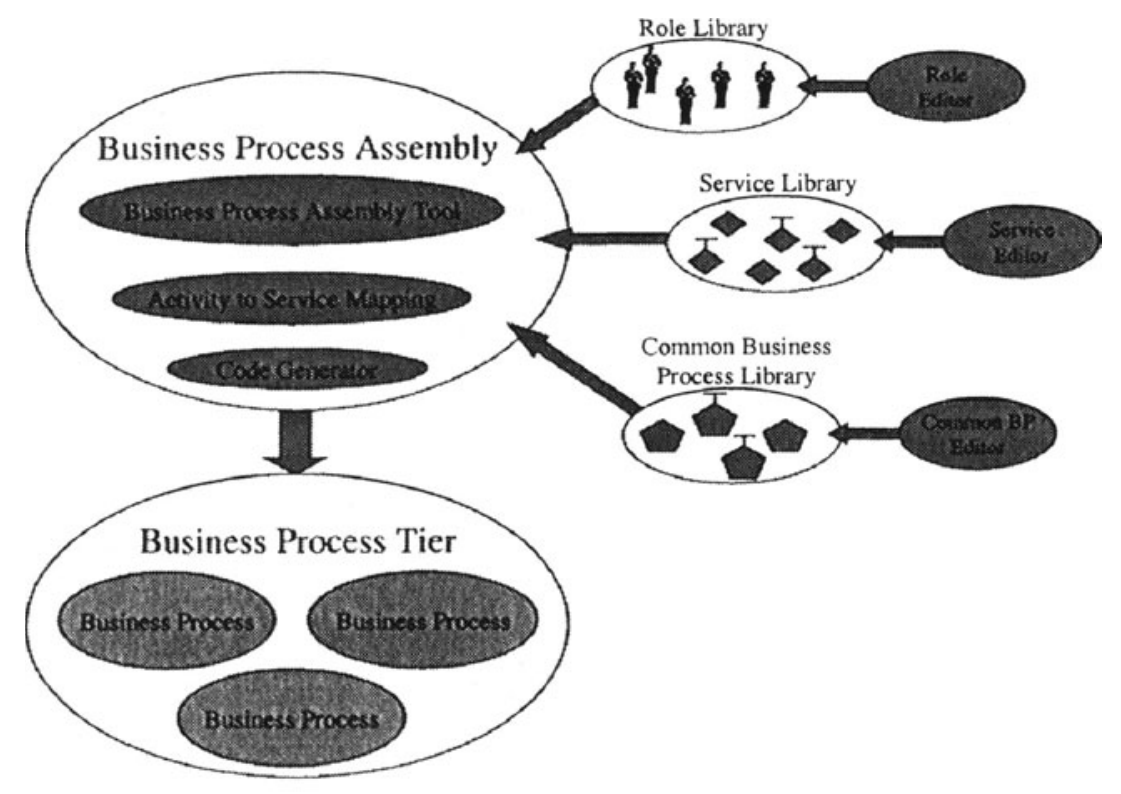

Figure 4 - Architecture for Automatically Program Code Generation

With the Business Process Assembly Tool the business processes are modeled using one of the above discussed standard language. Therefore, different libraries are used:

- Role-Library to define the participating roles

- Service-Library to define available services

- Common Business Process Library to define reusable business processes

Additional editors (Role Editor, Service Editor, and Common Business Process Editor) are necessary for the library management (e.g. create new entries, edit existing entries, etc.). Before the Code Generator is able to generate the program 
code for the Business Process Tier, an Activity to Service Mapping is necessary to define which service (service name, service location, etc.) is used to realize the business process activity.

The Code Generator itself can be implemented in two different ways:

- Consuming the XML-based output of the business process assembly unit shown in Figure 4, a Workflow-Engine can walk through the business processes activity by activity. Instead of program code, the executable input for a Workflow-Engine is generated.

- Using an XSLT processor to transform the XML-based description of the business processes into program code. For example, Figure 5 shows the scenario to generate Java Class Files. XSLT (XSL Transformations) is an XML-based language standardized by W3C (XSLT, 1999). There are a number of free XSLT processors available.

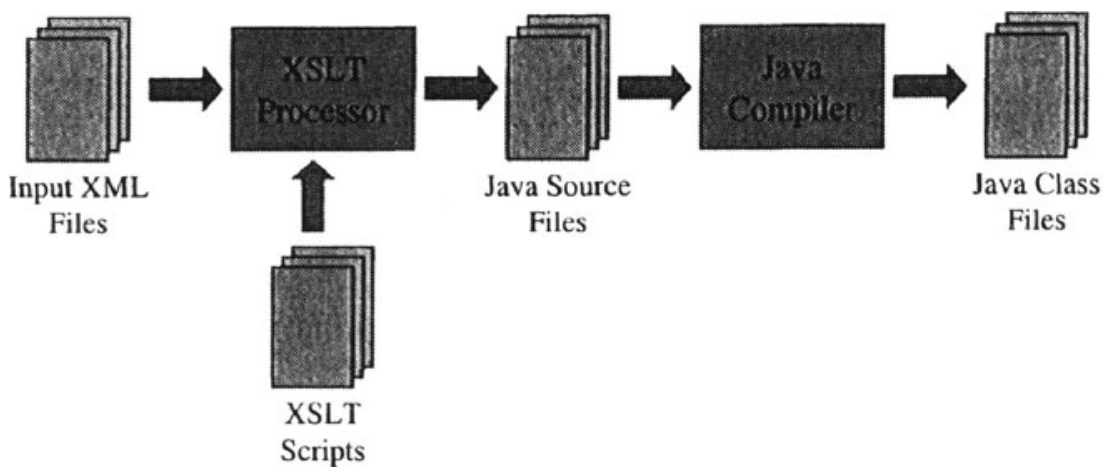

Figure 5 - Java Code Generation Using XSLT

In the near future a proof-of-concept prototype will be developed to show the usability of the proposed architecture for automatically program code generation.

\section{REFERENCES}

1. BPML (Business Process Modeling Language), Working Draft 0.4. Business Process Management Initiative. http://www.bpmi.org/. 8 March 2001.

2. ebXML Specifications. http://www.ebxml.org/specs/index.htm/. May 2001.

3. Fürst, Karl. „FLoCI-EE: Flexible Low-Cost Internet Extended Enterprise”. E-work and E-commerce. Editor: Brian Stanford-Smith and Enrica Chiozza. IOS Press, 2001; 769-775.

4. Linthicum, David S. Enterprise Application Integration. Addison Wesley, 2000.

5. SOAP (Simple Object Access Protocol) 1.1, W3C Note. World Wide Web Consortium (W3C). http://www.w3.org/TR/SOAP/. 8 May 2000.

6. Sun Microsystems: Sun Open Net Environment (Sun ONE). http://www.sun.com/sunonel. March 2002.

7. UDDI (Universal Description, Discovery, and Integration). http://www.uddi.org. March 2002.

8. WSDL (Web Services Description Language) 1.1, W3C Note. World Wide Web Consortium (W3C). http://www.w3.org/TR/wsdl. 15 March 2001.

9. WSFL (Web Services Flow Language) 1.0. IBM Software Group. http://www4.ibm.com/software/solutions/webservices/pdf/WSFL.pdf. May 2001.

10. XLANG. Microsoft Corporation. http://www.gotdotnet.com/team/xml wsspecs/xlang-c/default.htm. March 2002.

11. XSLT (XSL Transformations) 1.0, W3C Recommendation. World Wide Web Consortium (W3C). http://www.w3.org/TR/xslt. 16 November 1999. 